\title{
On a product formula for the Conley-Zehnder index of symplectic paths and its applications
}

\author{
Maurice De Gosson • Serge De Gosson • Paolo Piccione
}

Received: 20 June 2007 / Accepted: 5 February 2008 / Published online: 14 March 2008

(C) Springer Science+Business Media B.V. 2008

\begin{abstract}
Using invariance by fixed-endpoints homotopies and a generalized notion of symplectic Cayley transform, we prove a product formula for the Conley-Zehnder index of continuous paths with arbitrary endpoints in the symplectic group. We discuss two applications of the formula, to the metaplectic group and to periodic solutions of Hamiltonian systems.
\end{abstract}

Keywords Conley-Zehnder index

Mathematics Subject Classification (2000) $\quad 37 \mathrm{~B} 30 \cdot 81 \mathrm{~S} 10 \cdot 81 \mathrm{~S} 30$

\section{Introduction}

The theory of periodic Hamiltonian orbits plays a fundamental role in many active parts of both pure and applied mathematics. An object of choice for the qualitative and quantitative study of these orbits is the Conley-Zehnder index, introduced in [4], and whose theory has been further studied by many authors, see, e.g., $[1,16,23]$ and the references therein. Let us explain briefly what this index is about. Let $H \in C^{\infty}\left(\mathbb{R}^{2 n} \times \mathbb{R}, \mathbb{R}\right)$ be a time-dependent Hamiltonian, and denote by $\left(\mathcal{F}_{t}^{H}\right)$ the flow it determines; assume that $z_{0} \in \mathbb{R}^{2 n}$ is such that $\mathcal{F}_{1}^{H}(z)=z$, the mapping $t \longmapsto \mathcal{F}_{t}^{H}(z)$ is a 1-periodic orbit through $z_{0} \in \mathbb{R}^{2 n}$. Assume now that the Jacobian matrix $\Phi(z)=D \mathcal{F}_{1}(z)$ of $\mathcal{F}_{1}^{H}$ satisfies the non-degeneracy condition

Communicated by: J. Eichhorn (Greifswald, Germany).

M. De Gosson · S. De Gosson · P. Piccione ( $\varangle)$

Departamento De Matemática, Instituto De Matemática E Estatística, Universidade de São Paulo,

Rua Do Matão 1010, CEP 05508-090 Sao Paulo, SP, Brazil

e-mail: piccione.p@gmail.com

Present Address:

M. De Gosson

Max-Planck-Institut für Mathematik Pf. 7280, DE-53072, Bonn, Germany 


$$
\operatorname{det}(\Phi(z)-\mathrm{Id}) \neq 0
$$

Set now $z(t)=\mathcal{F}_{t}^{H}(z)$ and consider the linearized Hamiltonian system along $z$; its timeevolution is governed by the ordinary differential equation $\dot{u}=J D^{2} H(z, t) u$ whose flow consists of the symplectic matrices $\Phi_{t}=D \mathcal{F}_{t}^{H}(z)$. The path $\left.\left.\Sigma: t \longmapsto \Phi_{t}, t \in\right] 0,1\right]$ lies in the symplectic group $\operatorname{Sp}\left(\mathbb{R}^{2 n}, \omega\right)$; it starts from the identity and ends at $\Phi(z)$. If the non-degeneracy condition (1) holds one associates to $\Sigma$ an integer $i_{\mathrm{CZ}}(\Sigma)$, the ConleyZehnder index of the path $\Sigma$, and whose vocation is to give an algebraic count of the number of points $t_{j}$ in the interval ]0, 1[ for which $\Phi(z)-$ Id is not invertible. One should however be aware of the fact that condition (1) is very restrictive; in particular it is never satisfied in the simple case where $H$ is time-independent! The aim of this article is to give a general definition of the Conley-Zehnder index and to prove a formula for the index of the product of two symplectic paths, obtaining as a consequence a formula for the Conley-Zehnder index of an iterated periodic orbit. We observe that Cushman and Duistermaat [5] and Duistermaat [6] also have addressed the question of the index of the iteration of periodic orbits; the methods these authors use are however very different from ours. An extensive literature on the Maslov index and its iteration properties has been produced by Long and his collaborators (see for instance [19]), who obtained remarkable results on the multiplicity of periodic orbits of Hamiltonian systems. Relations between the Masov index and the spectral flow are studied in $[3,4,24]$.

In order to obtain the product formula in the general case of arbitrary endpoints, in this article we introduce the notion of generalized symplectic Cayley transform of a symplectomorphism. For each symplectomorphism $\psi$ whose spectrum does not contain 1 , we define a real-analytic diffeomorphism $\mathcal{C}_{\psi}$ between (an open subset of) the symplectic group and (an open subset of) the space of symmetric operators on a real finite dimensional Hilbert space. The classical symplectic Cayley transform is obtained when $\psi=-\mathrm{Id}$. The generalized Cayley transform is used to compute the correction term in the product formula (formula (10)) of symplectic paths.

This article is structured as follows: We begin by giving a working definition of the usual Conley-Zehnder index; we take the opportunity to recall a few basic definitions and results about some well-known objects from symplectic geometry such as the Hörmander, WallKashiwara, Maslov, and Leray indices; a good reference is the seminal paper [1] (see also $[17,21])$. The last part of this Section is devoted to the introduction and study of the main properties of a notion of symplectic Cayley transform which will be instrumental to our study of the product formula.

In Sect. 3 we state and prove the product formula for the Conley-Zehnder index in a very general setting in terms of the Hörmander index; in the special case where both paths are non-degenerate this formula can be restated very simply using the symplectic Cayley transform previously defined. The basic argument employed in that the proof of the product formula uses a homotopy properties of paths in topological groups

In Sect. 4 we pursue our study of the product formula and discuss the problem of the calculation of the index, an orbit which is iterated an arbitrary number of times; we obtain a number of precise estimates. These results aim at applications in the theory of periodic Hamiltonian orbits and at applications to spectral flow formulae and Morse theory (see [4,24, 25]).

Finally, in Sect. 5 we apply our results to the Weyl representation of metaplectic operators; this question is of fundamental importance in the study of the semiclassical quantization of non-integrable Hamiltonian systems (Gutzwiller's theory, see [15,20,22]); in particular we improve previous results [12]. 


\section{Preliminaries on Maslov, Conley-Zehnder, Kashiwara, Leray and Hörmander's indexes}

Let $V$ be a finite dimensional real vector space. $\mathrm{By}_{\mathrm{sym}}(V)$ we mean the space of all symmetric bilinear forms $B: V \times V \rightarrow \mathbb{R}$ on $V$; we will always identify a bilinear form $B: V \times V \rightarrow \mathbb{R}$ with the linear operator $V \ni v \mapsto B(v, \cdot) \in V^{*}$, that will be denoted by the same symbol $B$. For $B \in \mathrm{B}_{\text {sym }}(V)$, we denote by $\mathrm{n}_{-}(B), \mathrm{n}_{+}(B)$, respectively, the index and the coindex of $B$. The signature of $B$ is the difference $\operatorname{sign}(B)=\mathrm{n}_{-}(B)-\mathrm{n}_{+}(B)$. A bilinear form $B$ will be called nondegenerate if the linear map $V \ni v \mapsto B(v, \cdot) \in V^{*}$ is an isomorphism.

A symplectic form on $V$ is a nondegenerate antisymmetric bilinear form $\omega: V \times V \rightarrow \mathbb{R}$; the standard example of a symplectic space is $V=\mathbb{R}^{n} \oplus \mathbb{R}^{n *}$ endowed with the canonical symplectic form:

$$
\omega_{0}((v, \alpha),(w, \beta))=\beta(v)-\alpha(w), \quad v, w \in \mathbb{R}^{n}, \alpha, \beta \in \mathbb{R}^{n *} .
$$

The symplectic group $\operatorname{Sp}(V, \omega)$ is the closed subgroup of $\mathrm{GL}(V)$ consisting of those linear maps on $V$ that preserve $\omega$.

Given a symplectic space $(V, \omega)$, with $\operatorname{dim}(V)=2 n$, a Lagrangian subspace of $V$ is an $n$-dimensional subspace $L \subset V$ on which $\omega$ vanishes. The set of all Lagrangian subspaces of $V$, denoted by $\Lambda=\Lambda(V, \omega)$, has the structure of a compact, real-analytic submanifold of the Grassmannian of all $n$-dimensional subspaces of $V$. The dimension of $\Lambda$ equals $\frac{1}{2} n(n+1)$, and a real-analytic atlas on $\Lambda$ is given as follows.

For all $L \in \Lambda$ and $k \in\{0, \ldots, n\}$, let $\Lambda_{0}(L)$ denote the set of all Lagrangian subspaces that are transverse to $L$, which is a dense open subset of $\Lambda$. Given a pair $L_{0}, L_{1} \in \Lambda$ of complementary Lagrangians, i.e., $L_{0} \cap L_{1}=\{0\}$, then one defines a map:

$$
\varphi_{L_{0}, L_{1}}: \Lambda_{0}\left(L_{1}\right) \longrightarrow \mathrm{B}_{\text {sym }}\left(L_{0}\right)
$$

as follows. Any Lagrangian $L \in \Lambda_{0}\left(L_{1}\right)$ is the graph of a unique linear map $T: L_{0} \rightarrow L_{1}$; then, $\varphi_{L_{0}, L_{1}}$ is defined to be the restriction of the bilinear map $\omega(T \cdot, \cdot)$ to $L_{0} \times L_{0}$. It is easy to see that, due to the fact that $L$ is Lagrangian, such bilinear map is symmetric.

Given $L_{1} \in \Lambda$ and $L_{0}, L \in \Lambda_{0}\left(L_{1}\right)$, the bilinear forms $\varphi_{L_{0}, L_{1}}(L) \in \mathrm{B}_{\mathrm{sym}}\left(L_{0}\right)$ and $\varphi_{L, L_{1}}\left(L_{0}\right) \in \mathrm{B}_{\text {sym }}(L)$ are related by the identity:

$$
\eta^{*} \varphi_{L, L_{1}}\left(L_{0}\right)=-\varphi_{L_{0}, L_{1}}(L),
$$

where $\eta: L_{0} \rightarrow L$ is the isomorphism given by the restriction to $L_{0}$ of the projection $\pi^{L}: L \oplus L_{1} \cong V \rightarrow L$. In particular:

$$
\operatorname{sign}\left(\varphi_{L, L_{1}}\left(L_{0}\right)\right)=-\operatorname{sign}\left(\varphi_{L_{0}, L_{1}}(L)\right) .
$$

We need another identity relating the charts on $\Lambda$; assume that $L_{0}, L_{1}, L, L^{\prime}$ are Lagrangian subspaces of $V$, with $L_{0}, L, L^{\prime}$ transversal to $L_{1}$. Then:

$$
\varphi_{L, L_{1}}\left(L^{\prime}\right)=\eta_{0}^{*}\left(\varphi_{L_{0}, L_{1}}\left(L^{\prime}\right)-\varphi_{L_{0}, L_{1}}(L)\right),
$$

where $\eta_{0}: L \rightarrow L_{0}$ is the isomorphism given by the restriction to $L$ of the projection $L_{0} \oplus L_{1} \cong V \rightarrow L_{0}$. Hence:

$$
\operatorname{sign}\left(\varphi_{L, L_{1}}\left(L^{\prime}\right)\right)=\operatorname{sign}\left(\varphi_{L_{0}, L_{1}}\left(L^{\prime}\right)-\varphi_{L_{0}, L_{1}}(L)\right) .
$$

Given Lagrangians $L_{0}, L_{1} \subset V$ with $L_{0} \cap L_{1}=\{0\}$, then there exists a symplectic isomorphism (symplectomorphism) $\phi: V \rightarrow \mathbb{R}^{n} \oplus \mathbb{R}^{n *}$ (i.e., the pull-back $\phi^{*} \omega_{0}$ coincides with $\omega)$ such that $\phi\left(L_{0}\right)=\{0\} \oplus \mathbb{R}^{n *}$ and $\phi\left(L_{1}\right)=\mathbb{R}^{n} \oplus\{0\}$. 
2.1 Maslov index, Wall-Kashiwara's index and Leray index

Denote by $\pi(\Lambda)$ the fundamental groupoid of $\Lambda$, i.e., the set of fixed-endpoints homotopy classes $[\gamma]$ of continuous paths $\gamma$ in $\Lambda$, endowed with the partial operation of concatenation $\diamond$. For all $L_{0} \in \Lambda$, there exists a unique $\frac{1}{2} \mathbb{Z}$-valued groupoid homomorphism $\mu_{L_{0}}$ on $\pi(\Lambda)$ such that:

$$
\mu_{L_{0}}([\gamma])=\frac{1}{2} \operatorname{sign}\left(\varphi_{L_{0}, L_{1}}(\gamma(1))\right)-\frac{1}{2} \operatorname{sign}\left(\varphi_{L_{0}, L_{1}}(\gamma(0))\right)
$$

for all continuous curve $\gamma:[0,1] \rightarrow \Lambda_{0}\left(L_{1}\right)$ and for all $L_{1} \in \Lambda_{0}\left(L_{0}\right)$.

Definition 2.1 The map $\mu_{L_{0}}: \pi(\Lambda) \rightarrow \frac{1}{2} \mathbb{Z}$ is called the $L_{0}$-Maslov index.

The Maslov index has the following property (symplectic invariance): if $\phi:(V, \omega) \rightarrow$ $\left(V^{\prime}, \omega^{\prime}\right)$ is a symplectomorphism and $\gamma:[a, b] \rightarrow \Lambda(V, \omega)$ is continuous, then:

$$
\mu_{L_{0}}(\gamma)=\mu_{\phi\left(L_{0}\right)}(\phi \circ \gamma) \text {. }
$$

Moreover, replacing the symplectic form $\omega$ by $-\omega$ produces a change in the sign of $\mu_{L_{0}}$.

Let $\left(L_{0}, L_{1}, L_{2}\right)$ be a triple of elements of $\Lambda$; the Wall-Kashiwara index (see [1,26]) of that triple is the signature $\tau\left(L_{0}, L_{1}, L_{2}\right)$ of the quadratic form

$$
L_{0} \oplus L_{1} \oplus L_{2} \ni\left(z_{0}, z_{1}, z_{2}\right) \longmapsto \omega\left(z_{0}, z_{1}\right)+\omega\left(z_{1}, z_{2}\right)+\omega\left(z_{2}, z_{0}\right) \in \mathbb{R} .
$$

It is a $\operatorname{Sp}(V, \omega)$-invariant totally antisymmetric 2-cocycle on $\Lambda$. Let $\pi: \Lambda_{\infty} \longrightarrow \Lambda$ be the universal covering of $\Lambda$; the Leray index on $\Lambda_{\infty}$ is the unique $\mathbb{Z}$-valued 1-cochain $\mu$ on $\Lambda_{\infty}$ which is locally constant on $\left\{\left(L_{\infty}, L_{\infty}^{\prime}\right): \pi\left(L_{\infty}\right) \cap \pi\left(L_{\infty}^{\prime}\right)=0\right\}$ and such that $\partial \mu=\pi^{*} \tau$ ( $\partial$ the Čech coboundary operator). It is a symplectic invariant, in the sense that:

$$
\mu\left(\Phi_{\infty} L_{\infty}, \Phi_{\infty} L_{\infty}^{\prime}\right)=\mu\left(L_{\infty}, L_{\infty}^{\prime}\right)
$$

for all $\Phi_{\infty} \in \operatorname{Sp}_{\infty}(V, \omega)$ (the universal covering group of $\operatorname{Sp}(V, \omega)$ ). The Leray and Maslov indices are related in the following way: identifying $L_{\infty} \in \Lambda_{\infty}$ with the fixed-endpoints homotopy classes of continuous paths $\gamma$ joining $L_{0}$ to $L$, we have (see [11])

$$
\mu_{L_{0}}([\gamma])=\frac{1}{2}\left[\mu\left(\gamma(1)_{\infty}, L_{0, \infty}\right)-\mu\left(\gamma(0)_{\infty}, L_{0, \infty}\right)\right]
$$

where $L_{0, \infty}$ is the homotopy class of any loop through $L_{0}, \gamma(0)_{\infty}$ the homotopy class of any path $\gamma_{0}$ joining $L_{0}$ to $\gamma(0)$, and $\gamma(1)_{\infty}$ that of the concatenation $\gamma_{0} \diamond \gamma$. In view of the cochain relation $\partial \mu=\pi^{*} \tau$ this formula can be rewritten

$$
\mu_{L_{0}}([\gamma])=\frac{1}{2}\left[\mu\left(\gamma(1)_{\infty}, \gamma(0)_{\infty}\right)+\tau\left(L_{0}, \gamma(0), \gamma(1)\right)\right]
$$

(See Cappell et al. [1] for a comparative study of Leray and related indexes).

\subsection{Hörmander's index}

Given four Lagrangians $L_{0}, L_{1}, L_{0}^{\prime}, L_{1}^{\prime} \in \Lambda$ and any continuous curve $\gamma:[a, b] \rightarrow \Lambda$ such that $\gamma(a)=L_{0}^{\prime}$ and $\gamma(b)=L_{1}^{\prime}$, then the value of the quantity $\mu_{L_{1}}(\gamma)-\mu_{L_{0}}(\gamma)$ does not depend on the choice of $\gamma$.

Definition 2.2 Given $L_{0}, L_{1}, L_{0}^{\prime}, L_{1}^{\prime} \in \Lambda$, the Hörmander index $\mathfrak{q}\left(L_{0}, L_{1} ; L_{0}^{\prime}, L_{1}^{\prime}\right)$ is the half-integer number $\mu_{L_{1}}(\gamma)-\mu_{L_{0}}(\gamma)$, where $\gamma:[a, b] \rightarrow \Lambda$ is any continuous curve with $\gamma(a)=L_{0}^{\prime}$ and $\gamma(b)=L_{1}^{\prime}$. 
It follows from (7), the property $\partial \mu=\pi^{*} \tau$, and the fact that $\mu\left(L_{\infty}, L_{\infty}^{\prime}\right)$ is independent of the choice of base point in $\Lambda$ that we have

$$
\begin{gathered}
-\mathfrak{q}\left(L_{0}, L_{1} ; \gamma(0), \gamma(1)\right)=\mu_{L_{0}}([\gamma])-\mu_{L_{1}}([\gamma]) \\
=\frac{1}{2}\left[\tau\left(L_{0}, \gamma(0), \gamma(1)\right)-\tau\left(L_{1}, \gamma(0), \gamma(1)\right)\right] .
\end{gathered}
$$

\subsection{The Conley-Zehnder index}

Given a symplectic space $(V, \omega)$, consider the direct sum $V^{2}=V \oplus V$, endowed with the symplectic form $\omega^{2}=\omega \oplus(-\omega)$, defined by:

$$
\omega^{2}\left(\left(v_{1}, v_{2}\right),\left(w_{1}, w_{2}\right)\right)=\omega\left(v_{1}, v_{2}\right)-\omega\left(w_{1}, w_{2}\right), \quad v_{1}, v_{2}, w_{1}, w_{2} \in V .
$$

Given a linear operator $T: V \rightarrow V$, we will denote by $\operatorname{Gr}(T) \subset V^{2}$ its graph. Let $\Delta \subset V^{2}$ denote the diagonal; if $\Phi \in \operatorname{Sp}(V, \omega)$, then $\operatorname{Gr}(\Phi)=(\operatorname{Id} \oplus \Phi)[\Delta] \in \Lambda\left(V^{2}, \omega^{2}\right)$; in particular $\Delta=\mathrm{Gr}(\mathrm{Id})$ and $\Delta^{o}=\{(v,-v): v \in V\}=\mathrm{Gr}(-\mathrm{Id})$ are Lagrangian subspaces of $V^{2}$. If $\Phi_{1}, \Phi_{2} \in \operatorname{Sp}(V, \omega)$, then $\Phi_{1} \oplus \Phi_{2}: V^{2} \rightarrow V^{2}$ belongs to $\operatorname{Sp}\left(V^{2}, \omega^{2}\right)$.

Definition 2.3 Given a continuous curve $\Phi$ in $\operatorname{Sp}(V, \omega)$, the Conley-Zehnder index $\mathrm{i}_{\mathrm{CZ}}(\Phi)$ of $\Phi$ is the $\Delta$-Maslov index of the curve $t \mapsto \operatorname{Gr}(\Phi(t)) \in \Lambda\left(V^{2}, \omega^{2}\right)$ :

$$
\mathfrak{i}_{\mathrm{CZ}}(\Phi):=\mu_{\Delta}(t \mapsto \operatorname{Gr}(\Phi(t))) .
$$

The above is one of the possible definitions of the notion of Conley-Zehnder index (see $[4,23,25])$. The Conley-Zehnder index is additive by concatenation and invariant by fixed endpoint homotopies.

Consider the map $\mathcal{A}: V^{2} \rightarrow V^{2}$ given by $\mathcal{A}\left(v_{1}, v_{2}\right)=\left(v_{2}, v_{1}\right) ; \mathcal{A}$ is an antisymplectomorphism of $\left(V^{2}, \omega^{2}\right)$, i.e., $\mathcal{A}^{*} \omega^{2}=-\omega^{2}$. Clearly, $\mathcal{A}=\mathcal{A}^{-1}$ and $\mathcal{A}[\Delta]=\Delta$; more generally, if $S: V \rightarrow V$ is a bijection, then $\mathcal{A}[\operatorname{Gr}(S)]=\operatorname{Gr}\left(S^{-1}\right)$. It follows that, given a continuous path $\Phi:[a, b] \rightarrow \operatorname{Sp}(V, \omega)$, one has:

$$
\begin{aligned}
\mathfrak{i}_{\mathrm{CZ}}\left(\Phi^{-1}\right) & =\mu_{\Delta}\left(t \mapsto \operatorname{Gr}\left(\Phi^{-1}\right)\right)=\mu_{\Delta}(t \mapsto \mathcal{A}[\operatorname{Gr}(\Phi(t))]) \\
& =-\mu_{\mathcal{A}[\Delta]}(t \mapsto \operatorname{Gr}(\Phi(t)))=-\mu_{\Delta}(t \mapsto \operatorname{Gr}(\Phi(t)))=-\mathfrak{i}_{C Z}(\Phi) .
\end{aligned}
$$

Moreover, given four Lagrangians $L_{0}, L_{1}, L_{0}^{\prime}, L_{1}^{\prime} \in \Lambda$ :

$$
\mathfrak{q}\left(\mathcal{A}\left[L_{0}\right], \mathcal{A}\left[L_{1}\right] ; \mathcal{A}\left[L_{0}^{\prime}\right], \mathcal{A}\left[L_{1}^{\prime}\right]\right)=-\mathfrak{q}\left(L_{0}, L_{1} ; L_{0}^{\prime}, L_{1}^{\prime}\right) .
$$

Lemma 2.4 Let $\Phi:[a, b] \rightarrow \operatorname{Sp}(V, \omega)$ be a continuous curve, and let $\psi_{*} \in \operatorname{Sp}(V, \omega)$ be fixed. Denote by $\psi_{*} \cdot \Phi$ and by $\Phi \cdot \psi_{*}$ the continuous curves in $\operatorname{Sp}(V, \omega)$ given by $t \mapsto \psi_{*} \cdot \Phi(t)$ and $t \mapsto \Phi(t) \cdot \psi_{*}$, respectively. Then:

$$
\mathfrak{i}_{\mathrm{CZ}}\left(\psi_{*} \cdot \Phi\right)=\mathfrak{i}_{\mathrm{CZ}}\left(\Phi \cdot \psi_{*}\right)=\mathfrak{i}_{\mathrm{CZ}}(\Phi)+\mathfrak{q}\left(\Delta, \operatorname{Gr}\left(\psi_{*}^{-1}\right) ; \operatorname{Gr}(\Phi(a)), \operatorname{Gr}(\Phi(b))\right) .
$$

Proof A direct computation, as follows:

$$
\begin{aligned}
\mathfrak{i}_{\mathrm{CZ}}\left(\psi_{*} \cdot \Phi\right)= & \left.\mu_{\Delta}\left(t \mapsto \operatorname{Gr}\left(\psi_{*} \Phi(t)\right)\right)=\mu_{\Delta}\left(t \mapsto\left(\operatorname{Id} \oplus \psi_{*}\right)(\operatorname{Id} \oplus \Phi(t))\right)[\Delta]\right) \\
& \left.\stackrel{\text { symplectic invariance }}{=} \mu_{\left(\operatorname{Id} \oplus \psi_{*}^{-1}\right)[\Delta]}(t \mapsto(\operatorname{Id} \oplus \Phi(t)))[\Delta]\right) \\
= & \left.\mu_{\Delta}(t \mapsto(\operatorname{Id} \oplus \Phi(t)))[\Delta]\right)+\mathfrak{q}\left(\Delta,\left(\operatorname{Id} \oplus \psi_{*}^{-1}\right)[\Delta] ; \operatorname{Gr}(\Phi(a)), \operatorname{Gr}(\Phi(b))\right) \\
= & \mathfrak{i}_{\mathrm{CZ}}(\Phi)+\mathfrak{q}\left(\Delta, \operatorname{Gr}\left(\psi_{*}^{-1}\right) ; \operatorname{Gr}(\Phi(a)), \operatorname{Gr}(\Phi(b))\right) .
\end{aligned}
$$


Similarly,

$$
\begin{aligned}
\mathfrak{i}_{\mathrm{CZ}}\left(\Phi \cdot \psi_{*}\right) & =-\mathfrak{i}_{\mathrm{CZ}}\left(\psi_{*}^{-1} \cdot \Phi^{-1}\right) \\
& =-\left[\mathfrak{i}_{\mathrm{CZ}}\left(\Phi^{-1}\right)+\mathfrak{q}\left(\Delta, \operatorname{Gr}\left(\psi_{*}\right) ; \operatorname{Gr}\left(\Phi(a)^{-1}\right), \operatorname{Gr}\left(\Phi(b)^{-1}\right)\right)\right] \\
& =\mathfrak{i}_{\mathrm{CZ}}(\Phi)+\mathfrak{q}\left(\mathcal{A}[\Delta], \mathcal{A}\left[\operatorname{Gr}\left(\psi_{*}\right)\right] ; \mathcal{A}\left[\operatorname{Gr}\left(\Phi(a)^{-1}\right)\right], \mathcal{A}\left[\operatorname{Gr}\left(\Phi(b)^{-1}\right)\right]\right) \\
& =\mathfrak{i}_{\mathrm{CZ}}(\Phi)+\mathfrak{q}\left(\Delta, \operatorname{Gr}\left(\psi_{*}^{-1}\right) ; \operatorname{Gr}(\Phi(a)), \operatorname{Gr}(\Phi(b))\right) .
\end{aligned}
$$

\subsection{The symplectic Cayley transform}

Let us consider the two transverse Lagrangians $\Delta, \Delta^{o} \in \Lambda\left(V^{2}, \omega^{2}\right)$. Assume that $\Phi \in$ $\operatorname{Sp}(V, \omega)$ does not have the eigenvalue 1, i.e., that $\mathrm{Id}-\Phi$ is invertible or, equivalently, that $\operatorname{Gr}(\Phi)$ is transverse to $\Delta$.

An immediate computation shows that $\varphi_{\Delta^{o}, \Delta}(\operatorname{Gr}(\Phi))$ is identified with the symmetric bilinear form $2 \omega\left((\operatorname{Id}+\Phi)(\operatorname{Id}-\Phi)^{-1} \cdot, \cdot\right)$ on the vector space $V$. In particular, if $J$ is a complex structure on $V$ and $\langle\cdot, \cdot\rangle$ is a positive definite inner product on $V$ with $\langle J \cdot, \cdot\rangle=\omega$, then the linear operator $J(\operatorname{Id}+\Phi)(\operatorname{Id}-\Phi)^{-1}: V \rightarrow V$ is symmetric relatively to $\langle\cdot, \cdot\rangle$. In what follows, we will assume that the vector space $V$ is endowed with a positive definite inner product $\langle\cdot, \cdot\rangle$ and with a complex structure $J$ as above. We will denote by $\mathcal{L}_{\text {sa }}(V)$ the space of symmetric linear operators on $V$ and we will implicitly identify the spaces $\mathrm{B}_{\text {sym }}(V)$ and $\mathcal{L}_{\text {sa }}(V)$ by the obvious identification.

Definition 2.5 Given objects $V, \omega, J$ and $\langle\cdot, \cdot\rangle$ as above, and given $\Phi \in \operatorname{Sp}(V, \omega)$ with $(\mathrm{Id}-\Phi)$ invertible, then the symplectic Cayley transform $M_{\Phi}$ of $\Phi$ is the symmetric operator $\frac{1}{2} J(\mathrm{Id}+\Phi)(\mathrm{Id}-\Phi)^{-1}$.

One checks immediately the equality: $M_{\Phi^{-1}}=-M_{\Phi}$.

The notion of symplectic Cayley transform was originally introduced by Mehlig and Wilkinson (see [20]) and further studied in [12] and in [13]. We observe that the sign convention used in this paper differs from the original one.

In order to deal with symplectomorphisms whose spectrum contains 1 , we need to introduce a generalization of the notion of Cayley transform.

For a fixed $\psi \in \operatorname{Sp}(V, \omega)$, we denote by $\operatorname{Sp}_{\psi}(V, \omega)$ the dense open subset of $\operatorname{Sp}(V, \omega)$ consisting of those $\Phi \in \operatorname{Sp}(V, \omega)$ such that $\Phi-\psi$ is invertible. When $\psi=\operatorname{Id}$, it is customary to write $\operatorname{Sp}_{\text {Id }}(V, \omega)=\operatorname{Sp}_{0}(V, \omega)$.

Lemma 2.6 Let $\psi \in \operatorname{Sp}_{0}(V, \omega)$ be fixed. For all $\Phi \in \operatorname{Sp}_{\psi}(V, \omega)$, the linear operator

$$
\mathcal{C}_{\psi}(\Phi)=J(\psi-\mathrm{Id})(\Phi-\psi)^{-1}(\Phi-\mathrm{Id}): V \longrightarrow V
$$

is symmetric. Moreover, $\operatorname{Ker}\left(\mathcal{C}_{\psi}(\Phi)\right)=\operatorname{Ker}(\Phi-\mathrm{Id})$.

Proof The assumption $\psi \in \operatorname{Sp}_{0}(V, \omega)$ says that $\operatorname{Gr}(\psi) \in \Lambda\left(V^{2}, \omega^{2}\right)$ is transverse to $\Delta$; the assumption that $\Phi \in \operatorname{Sp}_{\psi}(V, \omega)$ says that $\operatorname{Gr}(\Phi) \in \Lambda\left(V^{2}, \omega^{2}\right)$ is transverse to $\operatorname{Gr}(\psi)$. A direct calculation shows that the symmetric bilinear form

$$
\varphi_{\Delta, \operatorname{Gr}(\psi)}(\operatorname{Gr}(\Phi)) \in \mathrm{B}_{\mathrm{sym}}(\Delta)
$$

can be identified (via the isomorphism $V \ni v \mapsto(v, v) \in \Delta$ ) with the bilinear form $\omega\left((\operatorname{Id}-\psi)(\Phi-\psi)^{-1}(\operatorname{Id}-\Phi) \cdot, \cdot\right)=\left\langle\mathcal{C}_{\psi}(\Phi) \cdot, \cdot\right\rangle$ on $V$. From this observation, the conclusion follows. The last statement in the thesis is obvious. 
Definition 2.7 Let $\psi \in \operatorname{Sp}_{0}(V, \omega)$ be fixed. The map $\mathcal{C}_{\psi}: \operatorname{Sp}_{\psi}(V, \omega) \rightarrow \mathcal{L}_{\text {sa }}(V)$ defined in Lemma 2.6 is called the $\psi$-Cayley transform.

\section{The product formula}

Let us start with a simple result on signature of the difference of symmetric bilinear forms (a similar result is proven in [23]):

Lemma 3.1 Let $V$ be a finite dimensional real vector space and let $U, Z \in \mathrm{B}_{\mathrm{sym}}(V)$ be nondegenerate symmetric bilinear forms on $V$ such that $U-Z$ is also nondegenerate. Then, $U^{-1}-Z^{-1}$ is nondegenerate and:

$$
\operatorname{sign}(Z)-\operatorname{sign}(U)=\operatorname{sign}\left(Z^{-1}-U^{-1}\right)-\operatorname{sign}(U-Z) .
$$

Proof Define the nondegenerate symmetric bilinear form $B \in \mathrm{B}_{\text {sym }}\left(V^{2}\right)$ by

$$
B\left(\left(a_{1}, b_{1}\right),\left(a_{2}, b_{2}\right)\right)=Z\left(a_{1}, a_{2}\right)-U\left(b_{1}, b_{2}\right) .
$$

Identifying $V$ with $\Delta$ by $v \mapsto(v, v)$, one computes easily $\left.B\right|_{\Delta}=Z-U$, which is nondegenerate. Denote by $\Delta^{\perp_{B}}$ the $B$-orthogonal complement of $\Delta$; identifying $V$ with $\Delta^{\perp_{B}}$ by $V \ni V \rightarrow\left(v, U^{-1} Z v\right) \in \Delta^{\perp_{B}}$, it is easily seen that $\left.B\right|_{\Delta^{\perp_{B}}}=Z\left(Z^{-1}-U^{-1}\right) Z$. The conclusion follows.

Proposition 3.2 Let $(V, \omega)$ be a symplectic vector space, and let $L, L^{\prime}, L_{0}, L_{1} \in \Lambda(V, \omega)$ be four Lagrangians, with $L, L^{\prime}, L_{1}$ transverse to $L_{0}$. Then:

$$
\mathfrak{q}\left(L_{0}, L ; L_{0}, L^{\prime}\right)=\frac{1}{2} \operatorname{sign}\left(\varphi_{L_{1}, L_{0}}(L)-\varphi_{L_{1}, L_{0}}\left(L^{\prime}\right)\right) .
$$

Proof Up to a symplectic isomorphism, we can assume $V=\mathbb{R}^{n} \oplus \mathbb{R}^{n *}$, with $\omega$ the canonical symplectic form $\omega_{0}$ (see (2)), $L_{0}=\{0\} \oplus \mathbb{R}^{n *}$ and $L_{1}=\mathbb{R}^{n} \oplus\{0\}$; transversality of $L$ and $L^{\prime}$ with $L_{0}$ says that we can write $L=\operatorname{Gr}(T), L^{\prime}=\operatorname{Gr}\left(T^{\prime}\right)$, where $T, T^{\prime}: \mathbb{R}^{n} \rightarrow \mathbb{R}^{n *}$ are self-adjoint linear maps. By definition, the Hörmander's index $\mathfrak{q}\left(L_{0}, L ; L_{0}, L^{\prime}\right)$ is given by:

$$
\mathfrak{q}\left(L_{0}, L ; L_{0}, L^{\prime}\right)=\mu_{L}(\gamma)-\mu_{L_{0}}(\gamma),
$$

where $\gamma:[a, b] \rightarrow \Lambda$ is an arbitrary continuous curve with $\gamma(a)=L_{0}$ and $\gamma(b)=L^{\prime}$.

In order to compute the two Maslov indexes in the formula above, let us choose a Lagrangian $\widetilde{L}_{1}$ which is transverse simultaneously to the three Lagrangians $L_{0}, L$, and $L^{\prime}$. We can choose, for instance, $\widetilde{L}_{1}=\operatorname{Gr}(S)$, where $S: \mathbb{R}^{n} \rightarrow \mathbb{R}^{n *}$ is a self-adjoint linear operator; in this way, $\widetilde{L}_{1}$ is transverse to $L_{0}$. Transversality of $\widetilde{L}_{1}$ to $L$ and $L^{\prime}$ is equivalent to $T-S$ and $T^{\prime}-S$ being invertible. Since $L_{0}, L^{\prime} \in \Lambda_{0}\left(\widetilde{L}_{1}\right)$, and $\Lambda_{0}\left(\widetilde{L}_{1}\right)$ is arc-connected, then one can choose a curve $\gamma$ with the required properties whose image is contained in $\Lambda_{0}\left(\widetilde{L}_{1}\right)$. Using (5), we then get:

$$
\begin{aligned}
& \mathfrak{q}\left(L_{0}, L ; L_{0}, L^{\prime}\right) \\
& \quad=\frac{1}{2}\left[\operatorname{sign}\left(\varphi_{L, \widetilde{L}_{1}}\left(L^{\prime}\right)\right)-\operatorname{sign}\left(\varphi_{L, \widetilde{L}_{1}}\left(L_{0}\right)\right)-\operatorname{sign}\left(\varphi_{L_{0}, \widetilde{L}_{1}}\left(L^{\prime}\right)\right)+\operatorname{sign}\left(\varphi_{L_{0}, \widetilde{L}_{1}}\left(L_{0}\right)\right)\right] .
\end{aligned}
$$

A direct calculation gives:

- $\varphi_{L_{1}, L_{0}}(L) \cong T: \mathbb{R}^{n} \rightarrow \mathbb{R}^{n *}$

- $\varphi_{L_{1}, L_{0}}\left(L^{\prime}\right) \cong T^{\prime}: \mathbb{R}^{n} \rightarrow \mathbb{R}^{n *}$;

- $\varphi_{L_{0}, \widetilde{L}_{1}}\left(L_{0}\right)=0$;

- $\varphi_{L_{0}, \widetilde{L}_{1}}\left(L^{\prime}\right) \cong\left(T^{\prime}-S\right)^{-1}: \mathbb{R}^{n *} \rightarrow \mathbb{R}^{n}$; 
- $\varphi_{L, \widetilde{L}_{1}}\left(L_{0}\right)$ is identified ${ }^{\star}$ with the symmetric bilinear form on $\mathbb{R}^{n}$ given by the self-adjoint linear operator $S-T: \mathbb{R}^{n} \rightarrow \mathbb{R}^{n *}$

- $\varphi_{L, \widetilde{L}_{1}}\left(L^{\prime}\right)$ is identified with the symmetric bilinear form on $\mathbb{R}^{n}$ given by:

$$
(S-T)+(T-S)\left(S-T^{\prime}\right)^{-1}(S-T): \mathbb{R}^{n} \rightarrow \mathbb{R}^{n *} .
$$

Using Lemma 3.1 and keeping in mind that sign $\left((S-T)+(T-S)\left(S-T^{\prime}\right)^{-1}(S-T)\right)=$ sign $\left((S-T)^{-1}-\left(S-T^{\prime}\right)^{-1}\right)$, from the equalities above one obtains:

$$
\mathfrak{q}\left(L_{0}, L ; L_{0}, L^{\prime}\right)=\frac{1}{2} \operatorname{sign}\left(T-T^{\prime}\right),
$$

which concludes the proof.

Lemma 3.3 Given continuous paths $\Phi_{i}:[0,1] \rightarrow \mathrm{Sp}(V, \omega), i=1,2$, then the pointwise product $\Phi(t)=\Phi_{1}(t) \cdot \Phi_{2}(t)$ is fixed-endpoints homotopic to the concatenation $\widetilde{\Phi} \diamond \widetilde{\Phi}_{2}$, where $\widetilde{\Phi}_{i}:[0,1] \rightarrow \operatorname{Sp}(V, \omega)$ is given by:

$$
\widetilde{\Phi}_{1}(t)=\Phi_{1}(t) \cdot \Phi_{2}(0), \quad \widetilde{\Phi}_{2}(t)=\Phi_{1}(1) \cdot \Phi_{2}(t), \quad \forall t \in[0,1] .
$$

In particular, $\mathfrak{i}_{\mathrm{CZ}}(\Phi)=\mathfrak{i}_{\mathrm{CZ}}\left(\widetilde{\Phi}_{1}\right)+\mathfrak{i}_{\mathrm{CZ}}\left(\widetilde{\Phi}_{2}\right)$.

Proof The curve $\Phi_{1}$ is fixed-endpoints homotopic to the curve $\bar{\Phi}_{1}$ defined by:

$$
\bar{\Phi}_{1}(t)= \begin{cases}\Phi_{1}(2 t), & \text { if } t \in\left[0, \frac{1}{2}\right] ; \\ \Phi_{1}(1), & \text { if } t \in\left[\frac{1}{2}, 1\right],\end{cases}
$$

while $\Phi_{2}$ is fixed-endpoints homotopic to $\bar{\Phi}_{2}$, given by:

$$
\bar{\Phi}_{2}(t)= \begin{cases}\Phi_{2}(0), & \text { if } t \in\left[0, \frac{1}{2}\right] \\ \Phi_{2}(2 t-1), & \text { if } t \in\left[\frac{1}{2}, 1\right],\end{cases}
$$

hence the pointwise product $\Phi_{1} \cdot \Phi_{2}$ is fixed-endpoints homotopic to the pointwise product $\bar{\Phi}_{1} \cdot \bar{\Phi}_{2}$. Clearly, $\bar{\Phi}_{1} \cdot \bar{\Phi}_{2}=\widetilde{\Phi}_{1} \diamond \widetilde{\Phi}_{2}$.

An immediate application of Lemma 2.4 and Lemma 3.3 gives:

Corollary 3.4 Given continuous paths $\Phi_{i}:[0,1] \rightarrow \mathrm{Sp}(V, \omega), i=1,2$, then:

$$
\begin{aligned}
\mathfrak{i}_{\mathrm{CZ}}\left(\Phi_{1} \cdot \Phi_{2}\right)= & \mathfrak{i}_{\mathrm{CZ}}\left(\Phi_{1}\right)+\mathfrak{i}_{\mathrm{CZ}}\left(\Phi_{2}\right) \\
& +\mathfrak{q}\left(\Delta, \operatorname{Gr}\left(\Phi_{2}(0)^{-1}\right) ; \operatorname{Gr}\left(\Phi_{1}(0)\right), \operatorname{Gr}\left(\Phi_{1}(1)\right)\right) \\
& +\mathfrak{q}\left(\Delta, \operatorname{Gr}\left(\Phi_{1}(1)^{-1}\right) ; \operatorname{Gr}\left(\Phi_{2}(0)\right), \operatorname{Gr}\left(\Phi_{2}(1)\right)\right) .
\end{aligned}
$$

In particular, if $\Phi_{2}$ is a homotopically trivial loop starting at the identity of $\operatorname{Sp}(V, \omega)$, then $\mathfrak{i}_{\mathrm{CZ}}\left(\Phi_{1} \cdot \Phi_{2}\right)=\mathfrak{i}_{\mathrm{CZ}}\left(\Phi_{1}\right)$.

Let us now consider the case that the paths $\Phi_{1}$ and $\Phi_{2}$ start at the identity of $\operatorname{Sp}(V, \omega)$, in which case obviously $\operatorname{Gr}\left(\Phi_{1}(0)\right)=\operatorname{Gr}\left(\Phi_{2}(0)\right)=\Delta$, and the term

$$
\mathfrak{q}\left(\Delta, \operatorname{Gr}\left(\Phi_{2}(0)^{-1}\right) ; \operatorname{Gr}\left(\Phi_{1}(0)\right), \operatorname{Gr}\left(\Phi_{1}(1)\right)\right)=\mathfrak{q}\left(\Delta, \Delta ; \Delta, \operatorname{Gr}\left(\Phi_{1}(1)\right)\right)
$$

in equality (9) vanishes.

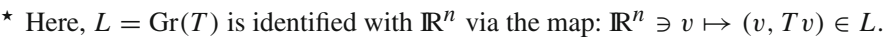


Corollary 3.5 Let $\Phi_{i}:[0,1] \rightarrow \operatorname{Sp}(V, \omega)$ be continuous paths with $\Phi_{1}(0)=\Phi_{2}(0)=\mathrm{Id}$ and with $\mathrm{Id}-\Phi_{1}(1), \mathrm{Id}-\Phi_{2}(1)$ invertible. Then:

$$
\mathfrak{i}_{\mathrm{CZ}}\left(\Phi_{1} \cdot \Phi_{2}\right)=\mathfrak{i}_{\mathrm{CZ}}\left(\Phi_{2} \cdot \Phi_{1}\right)=\mathfrak{i}_{\mathrm{CZ}}\left(\Phi_{1}\right)+\mathfrak{i}_{\mathrm{CZ}}\left(\Phi_{2}\right)-\frac{1}{2} \operatorname{sign}\left(M_{\Phi_{1}(1)}+M_{\Phi_{2}(1)}\right) .
$$

Proof Formula (10) follows easily from (9), using Proposition 3.2 applied to the symplectic space $\left(V^{2}, \omega^{2}\right)$ and to the Lagrangians $L_{0}=\Delta, L_{1}=\Delta^{o}, L=\operatorname{Gr}\left(\Phi_{1}(1)^{-1}\right)$ and $L^{\prime}=\operatorname{Gr}\left(\Phi_{2}(1)\right)$.

The result of Corollary 3.5 can be extended to the case of paths with arbitrary endpoints in the symplectic group, using our generalized notion of symplectic Cayley transform.

Proposition 3.6 Let $\phi_{1}, \phi_{2}, \psi \in \operatorname{Sp}(V, \omega)$ be fixed; assume that $\psi \in \operatorname{Sp}_{0}(V, \omega)$ and that $\phi_{1}, \phi_{2} \in \operatorname{Sp}_{\psi}(V, \omega)$. Then, the Hörmander index $\mathfrak{q}\left(\Delta, \operatorname{Gr}\left(\phi_{1}\right) ; \Delta, \operatorname{Gr}\left(\phi_{2}\right)\right)$ is given by:

$\mathfrak{q}\left(\Delta, \operatorname{Gr}\left(\phi_{1}\right) ; \Delta, \operatorname{Gr}\left(\phi_{2}\right)\right)=\frac{1}{2}\left[\operatorname{sign}\left(\mathcal{C}_{\psi}\left(\phi_{2}\right)-\mathcal{C}_{\psi}\left(\phi_{1}\right)\right)-\operatorname{sign}\left(\mathcal{C}_{\psi}\left(\phi_{2}\right)\right)+\operatorname{sign}\left(\mathcal{C}_{\psi}\left(\phi_{1}\right)\right)\right]$.

If $\phi_{1}, \phi_{2} \in \operatorname{Sp}_{0}(V, \omega)$, then:

$$
\mathfrak{q}\left(\Delta, \operatorname{Gr}\left(\phi_{1}\right) ; \Delta, \operatorname{Gr}\left(\phi_{2}\right)\right)=\frac{1}{2} \operatorname{sign}\left(\mathcal{C}_{\psi}\left(\phi_{1}\right)^{-1}-\mathcal{C}_{\psi}\left(\phi_{2}\right)^{-1}\right) .
$$

Proof By definition of Hörmander index, $\mathfrak{q}\left(\Delta, \operatorname{Gr}\left(\phi_{1}\right) ; \Delta, \operatorname{Gr}\left(\phi_{2}\right)\right)$ is given by:

$$
\mu_{\operatorname{Gr}\left(\phi_{1}\right)}(\gamma)-\mu_{\Delta}(\gamma)
$$

where $\gamma:[a, b] \rightarrow \Lambda\left(V^{2}, \omega^{2}\right)$ is any continuous curve with $\gamma(a)=\Delta$ and $\gamma(b)=\operatorname{Gr}\left(\phi_{2}\right)$. We can choose one such curve $\gamma$ whose image remains inside the set of Lagrangians of $\left(V^{2}, \omega^{2}\right)$ that are transversal to $\operatorname{Gr}(\psi)$. Then, by definition of Maslov index, we have:

$$
\begin{aligned}
\mu_{\operatorname{Gr}\left(\phi_{1}\right)}(\gamma)-\mu_{\Delta}(\gamma)= & \frac{1}{2}\left[\operatorname{sign}\left(\varphi_{\operatorname{Gr}\left(\phi_{1}\right), \operatorname{Gr}(\psi)}\left(\operatorname{Gr}\left(\phi_{2}\right)\right)\right)-\operatorname{sign}\left(\varphi_{\operatorname{Gr}\left(\phi_{1}\right), \operatorname{Gr}(\psi)}(\Delta)\right)\right] \\
& -\frac{1}{2}\left[\operatorname{sign}\left(\varphi_{\Delta, \operatorname{Gr}(\psi)}\left(\operatorname{Gr}\left(\phi_{2}\right)\right)\right)-\operatorname{sign}\left(\varphi_{\Delta, \operatorname{Gr}(\psi)}(\Delta)\right)\right] .
\end{aligned}
$$

The first equality in the thesis is now obtained easily, using the following:

- $\varphi_{\Delta, \operatorname{Gr}(\psi)}\left(\operatorname{Gr}\left(\phi_{2}\right)\right)=\mathcal{C}_{\psi}\left(\phi_{2}\right)$;

- $\operatorname{sign}\left(\varphi_{\operatorname{Gr}\left(\phi_{1}\right), \operatorname{Gr}(\psi)}(\Delta)\right) \stackrel{\text { by }(3)}{=}-\operatorname{sign}\left(\varphi_{\Delta, \operatorname{Gr}(\psi)}\left(\operatorname{Gr}\left(\phi_{1}\right)\right)\right)=-\operatorname{sign}\left(\mathcal{C}_{\psi}\left(\phi_{1}\right)\right)$;

- $\operatorname{sign}\left(\varphi_{\operatorname{Gr}\left(\phi_{1}\right), \operatorname{Gr}(\psi)}\left(\operatorname{Gr}\left(\phi_{2}\right)\right)\right) \stackrel{\text { by }(4)}{=} \operatorname{sign}\left(\varphi_{\Delta, \operatorname{Gr}(\psi)}\left(\operatorname{Gr}\left(\phi_{2}\right)\right)-\varphi_{\Delta, \operatorname{Gr}(\psi)}\left(\operatorname{Gr}\left(\phi_{1}\right)\right)\right)$

- $\varphi_{\Delta, \operatorname{Gr}(\psi)}(\Delta)=0$.$$
=\operatorname{sign}\left(\mathcal{C}_{\psi}\left(\phi_{2}\right)-\mathcal{C}_{\psi}\left(\phi_{1}\right)\right)
$$

Finally, if $\phi_{1}, \phi_{2} \in \operatorname{Sp}_{0}(V, \omega)$, i.e., if $\mathcal{C}_{\psi}\left(\phi_{1}\right)$ and $\mathcal{C}_{\psi}\left(\phi_{2}\right)$ are invertible, then, by Lemma 3.1:

$\operatorname{sign}\left(\mathcal{C}_{\psi}\left(\phi_{2}\right)-\mathcal{C}_{\psi}\left(\phi_{1}\right)\right)-\operatorname{sign}\left(\mathcal{C}_{\psi}\left(\phi_{2}\right)\right)+\operatorname{sign}\left(\mathcal{C}_{\psi}\left(\phi_{1}\right)\right)=\operatorname{sign}\left(\mathcal{C}_{\psi}\left(\phi_{1}\right)^{-1}-\mathcal{C}_{\psi}\left(\phi_{2}\right)^{-1}\right)$, which concludes the proof.

Corollary 3.7 Let $\Phi_{i}:[0,1] \rightarrow \operatorname{Sp}(V, \omega)$ be continuous paths with $\Phi_{1}(0)=\Phi_{2}(0)=\mathrm{Id}$, and let $\psi \in \operatorname{Sp}_{0}(V, \omega)$ be such that $\Phi_{1}(1), \Phi_{2}(1) \in \operatorname{Sp}_{\psi}(V, \omega)$. Then:

$$
\begin{aligned}
\mathfrak{i}_{\mathrm{CZ}}\left(\Phi_{1} \cdot \Phi_{2}\right)= & \mathfrak{i}_{\mathrm{CZ}}\left(\Phi_{1}\right)+\mathfrak{i}_{\mathrm{CZ}}\left(\Phi_{2}\right)+\frac{1}{2} \operatorname{sign}\left[\mathcal{C}_{\psi}\left(\Phi_{2}(1)\right)-\mathcal{C}_{\psi}\left(\Phi_{1}(1)\right)\right] \\
& \left.\left.-\frac{1}{2} \operatorname{sign}\left[\mathcal{C}_{\psi}\left(\Phi_{2}(1)\right)\right)\right]+\frac{1}{2} \operatorname{sign}\left[\mathcal{C}_{\psi}\left(\Phi_{1}(1)\right)\right)\right] .
\end{aligned}
$$

If $\Phi:[0,1] \rightarrow \operatorname{Sp}(V, \omega)$ is a continuous path, then:

$$
|| \mathfrak{i}_{\mathrm{CZ}}\left(\Phi^{N}\right)|-N| \mathfrak{i}_{\mathrm{CZ}}(\Phi)|| \leq \frac{1}{2} n(N-1),
$$

for all $N \geq 1$. 
Proof The proof of formula (11) is analogous to the proof of (10), where one replaces the chart $\varphi_{\Delta^{o}, \Delta}$ by $\varphi_{\Delta, \operatorname{Gr}(\psi)}$, using the result of Proposition 3.6 for the computation of the Hörmander index. The inequality in formula (12) is obtained readily from (11) using induction on $N$; for such induction argument one has to keep in mind the following observations.

- One has to use Cayley transform $\mathcal{C}_{\psi}$, where $\psi \in \operatorname{Sp}_{0}(V, \omega)$ is such that all the powers $\Phi(1)^{N}$ belong to $\operatorname{Sp}_{\psi}(V, \omega)$. The set of such $\psi$ 's is non empty, and in fact dense in $\operatorname{Sp}(V, \omega)$; namely, this set is the intersection of the countable family of dense open subsets $\operatorname{Sp}_{0}(V, \omega) \cap \operatorname{Sp}_{\Phi(1)^{j}}(V, \omega), j \geq 1$, and the claims follows from Baire's theorem.

- Given any pair $B_{1}, B_{2}$ of symmetric bilinear form on any $n$-dimensional real vector space, then

$$
\left|\operatorname{sign}\left(B_{2}-B_{1}\right)-\operatorname{sign}\left(B_{2}\right)-\operatorname{sign}\left(B_{1}\right)\right| \leq n .
$$

Namely, if $B_{1}$ and $B_{2}$ are nondegenerate, the claim follows immediately from Lemma 3.1; for the general case simply use an argument of density and continuity.

The inequality in formula (12) tells us that if $\left|\mathfrak{i}_{\mathrm{CZ}}(\Phi)\right| \geq \frac{n}{2}$, then $\left|\mathfrak{i}_{\mathrm{CZ}}\left(\Phi^{N}\right)\right|$ has a linear growth in $N$.

\section{Iteration formulae}

Let us now discuss the problem of determining the Conley-Zehnder index and the Maslov index of the iteration of a periodic solution of a Hamiltonian system.

Let $(\mathcal{M}, \varpi)$ be a $2 n$-dimensional symplectic manifold, and let $H: \mathcal{M} \times \mathbb{R} \rightarrow \mathbb{R}$ be a timedependent smooth Hamiltonian. Assume that $H$ is $T$-periodic in time, and that $z:[0, T] \rightarrow M$ is a solution of $H$ (i.e., $\dot{z}=\vec{H}(z)$ such that $z(0)=z(T)$, where $\vec{H}$ is the time-dependent Hamiltonian vector field, defined by $\varpi(\vec{H}, \cdot)=\mathrm{d} H)$. Then, the iterates $z^{(N)}$ of $z$, defined as the concatenation:

$$
z^{(N)}=\underbrace{z \diamond \cdots \diamond z}_{N \text {-times }}:[0, N T] \longrightarrow \mathcal{M}
$$

are also solutions of $H$. Assume that it is given a periodic symplectic trivialization of the tangent bundle of $\mathcal{M}$ along $z$ (i.e., of the pull-back $z^{*} T \mathcal{M}$ ), which consists of a smooth family $\Psi=\left\{\psi_{t}\right\}_{t \in[0, T]}$ of symplectomorphisms $\psi_{t}: T_{z(0)} \mathcal{M} \rightarrow T_{z(t)} \mathcal{M}$ with $\psi_{0}=\psi_{T}=$ Id. By a simple orientability argument, periodic symplectic trivializations along periodic solutions always exist. By the periodicity assumption, we have a smooth extension $\mathbb{R} \ni t \mapsto \psi_{t}$ by setting $\psi_{t+N T}=\psi_{t}$ for all $t \in[0, T]$.

Denote by $\mathcal{F}_{t, t^{\prime}}^{H}: \mathcal{M} \rightarrow \mathcal{M}$ the (maximal) flow of $\vec{H},{ }^{\star}$ i.e., $\mathcal{F}_{t, t^{\prime}}^{H}(p)=\gamma\left(t^{\prime}\right)$, where $\gamma$ is the unique integral curve of the time-dependent vector field $\vec{H}$ on $\mathcal{M}$ satisfying $\gamma(t)=p$. It is well known that for all $t, t^{\prime}$, the $\mathcal{F}_{t, t^{\prime}}^{H}$ is a symplectomorphism among open subsets of $\mathcal{M}$. Left composition with $\psi_{t}^{-1}$ gives a smooth map $\mathbb{R} \ni t \mapsto \Psi(t)=\psi_{t}^{-1} \circ \mathcal{F}_{0, t}^{H}(z(0))$ of linear symplectomorphisms of $T_{z(0)} \mathcal{M}$; clearly $X(t)=\Psi^{\prime}(t) \Psi(t)^{-1}$ lies in the Lie algebra $\operatorname{sp}\left(T_{z(0)} \mathcal{M}, \varpi_{z(0)}\right)$ of the symplectic group $\operatorname{Sp}\left(T_{z(0)} \mathcal{M}, \varpi_{z(0)}\right)$.

The linearized Hamilton equation along $z$ is the linear system

$$
v^{\prime}(t)=X(t) v(t)
$$

\footnotetext{
${ }_{\star}$ For our purposes, we will not be interested in questions of global existence of the flow $\mathcal{F}^{H}$.
} 
in $T_{z(0)} \mathcal{M}$; the fundamental solution of this linear system is a smooth symplectic path $\Phi$ : $\mathbb{R} \rightarrow \operatorname{Sp}\left(T_{z(0)} \mathcal{M}, \varpi_{z(0)}\right)$ that satisfies $\Phi(0)=\operatorname{Id}$ and $\Phi^{\prime}=X \Phi$.

Definition 4.1 The Conley-Zehnder index of the solution $z^{(1)}$ associated to the symplectic trivialization $\Psi$, denoted by $i_{\mathrm{CZ}}\left(z^{(1)}, \Psi\right)$, is the Conley-Zehnder of the path in $\operatorname{Sp}\left(T_{z(0)} \mathcal{M}, \varpi_{z(0)}\right)$ obtained by restriction of the fundamental solution $\Phi$ to the interval $[0, T]$.

Remark 4.2 It is known that, under suitable topological condition on the manifold $\mathcal{M}$ and on the loop $z$, the Conley-Zehnder index $i_{\mathrm{CZ}}\left(z^{(1)}, \Psi\right)$ will not depend on the choice of the trivialization $\Psi$. For instance, if $z: \mathbb{S}^{1} \rightarrow \mathcal{M}$ is homotopically trivial, i.e., if $z$ admits a continuous extension to the 2-disk $\mathbb{D}^{2}$, then one can choose trivializations $\Psi$ of $z^{*}(T \mathcal{M})$ that admit continuous extensions to $\mathbb{D}^{2}$. In this situation, if the first Chern class $c_{1}(\mathcal{M})$ has vanishing integral on every 2 -sphere of $\mathcal{M}$, then the Conley-Zehnder index of $z$ will not depend on $\Psi$. Namely, in this case any two trivializations in the required class differ by a loop in the symplectic group which is homotopically trivial which, by Corollary 3.4, does not alter the value of the Conley-Zehnder index.

Using the results of Sect. 3, we can estimate the Conley-Zehnder index of the iterated of a periodic Hamiltonian solution as follows:

Proposition 4.3 For all $N \geq 1$, define $\Phi_{(N)}:[0, N T] \rightarrow \operatorname{Sp}\left(T_{z(0)} \mathcal{M}, \varpi_{z(0)}\right)$ to be the restriction to the interval $[0, N T]$ of the fundamental solution $\Phi$ of (13). Then, $\Phi_{(N)}$ is fixed-endpoint homotopic to the $N$ th power of $\Phi_{(1)}$.

Proof Use the group law $\Phi(t+T)=\Phi(t) \cdot \Phi(T)$, and argue as in the proof of Lemma 3.3.

Corollary 4.4 In the above notations, the following inequality holds:

$$
\left|\mathfrak{i}_{\mathrm{CZ}}\left(z^{(N)}, \Psi\right)-N \cdot \mathfrak{i}_{\mathrm{CZ}}\left(z^{(1)}, \Psi\right)\right| \leq \frac{1}{2} n(N-1) .
$$

In particular, $\left|\mathfrak{i}_{\mathrm{CZ}}\left(z^{(N)}, \Psi\right)\right|$ and $\left|\mu_{L_{0}}\left(z^{(N)}, \Psi\right)\right|$ have sublinear growth in $N$; moreover, if $\left|\mathfrak{i}_{\mathrm{CZ}}\left(z^{(1)}, \Psi\right)\right|>\frac{1}{2} n\left(\right.$ resp. if $\left.\mu_{L_{0}}\left(z^{(1)}, \Psi\right)>\frac{7}{2} n\right)$, then $\mathfrak{i}_{\mathrm{CZ}}\left(z^{(N)}, \Psi\right)\left(\right.$ resp., $\left.\mu_{L_{0}}\left(z^{(N)}, \Psi\right)\right)$ has linear growth in $N$.

Proof Follows immediately from Corollary 3.7 and Proposition 4.3.

\section{The Weyl representation of $\operatorname{Mp}(2 n, \mathbb{R})$}

\subsection{The metaplectic group}

We say that a quadratic form $W: \mathbb{R}^{n} \times \mathbb{R}^{n} \longrightarrow \mathbb{R}$ is "non-degenerate" if it can be written

$$
W\left(x, x^{\prime}\right)=\frac{1}{2}\langle P x, x\rangle-\left\langle K x, x^{\prime}\right\rangle+\frac{1}{2}\left\langle Q x^{\prime}, x^{\prime}\right\rangle
$$

where $P$ and $Q$ are symmetric and $K$ invertible. The data of such a quadratic form determine a symplectomorphism $\Phi_{W} \in \operatorname{Sp}(2 n \mathbb{R})$, whose matrix in the canonical basis of $\mathbb{R}^{2 n}$ is written in $n \times n$ blocks as:

$$
\left(\begin{array}{cc}
K^{-1} Q & K^{-1} \\
P K^{-1} Q-K^{T} & K^{-1} P
\end{array}\right) .
$$

Set $L_{0}=\{0\} \times \mathbb{R}^{n}$; the symplectomorphism $\Phi_{W}$ can be characterized by the properties: 
- $\Phi_{W}\left(L_{0}\right) \cap L_{0}=\{0\}$

- $\quad$ setting $z=(x, p), z^{\prime}=\left(x^{\prime}, p^{\prime}\right) \in \mathbb{R}^{2 n}$, then $z=\Phi_{W}\left(z^{\prime}\right)$ if and only if $p=\partial_{x} W\left(x, x^{\prime}\right)$ and $p^{\prime}=-\partial_{x^{\prime}} W\left(x, x^{\prime}\right)$.

Definition 5.1 $\Phi_{W}$ is the free symplectic automorphism determined by the generating function $W$.

Let $\mathcal{S}\left(\mathbb{R}^{n}\right)$ be the Schwartz space of rapidly decreasing funcions on $\mathbb{R}^{n}$. We associate to $\Phi_{W}$ the Fourier integral operator $\widehat{\Phi}_{W, m}: \mathcal{S}\left(\mathbb{R}^{n}\right) \longrightarrow \mathcal{S}\left(\mathbb{R}^{n}\right)$ defined by

$$
\widehat{\Phi}_{W, m} f(x)=(2 \pi i)^{-n / 2} \Delta(W) \int e^{i W\left(x, x^{\prime}\right)} f\left(x^{\prime}\right) d^{n} x^{\prime}
$$

where $\Delta(W)=i^{m} \sqrt{|\operatorname{det} K|}$ and $m$ corresponds to a choice of $\arg \operatorname{det} K$ through

$$
m \pi=\arg (\operatorname{det} K) \bmod 4 \pi
$$

(for each $K$ there are thus two choices of $m$ modulo 4 ). The operators $\widehat{\Phi}_{W, m}$ extend by continuity to unitary operators on $L^{2}\left(\mathbb{R}^{n}\right)$; the inverse of $\widehat{\Phi}_{W, m}$ is $\widehat{\Phi}_{W^{*}, m^{*}}$ with $W^{*}\left(x, x^{\prime}\right)=$ $-W\left(x^{\prime}, x\right)$ and $m^{*}=n-m$. These operators thus generate a group of unitary operators on $L^{2}\left(\mathbb{R}^{n}\right)$, the metaplectic group $\operatorname{Mp}(2 n, \mathbb{R})$, which is a double cover of $\operatorname{Sp}(2 n, \mathbb{R})$; the projection $\pi^{\mathrm{Mp}}: \operatorname{Mp}(2 n, \mathbb{R}) \longrightarrow \mathrm{Sp}(2 n, \mathbb{R})$ is unambiguously determined by the condition $\pi^{\mathrm{Mp}}\left(\widehat{\Phi}_{W, m}\right)=\Phi_{W}$. We have (see [12]):

Proposition 5.2 For every $\widehat{\Phi} \in \mathrm{Mp}(2 n, \mathbb{R})$ there exist two generating functions $W$ and $W^{\prime}$ and integers $m, m^{\prime}$ such that $\widehat{\Phi}=\widehat{\Phi}_{W, m} \widehat{\Phi}_{W^{\prime}, m^{\prime}}$ and $\Phi_{W}, \Phi_{W^{\prime}} \in \operatorname{Sp}_{0}(2 n, \mathbb{R})$; the condition $\Phi_{W} \in \mathrm{Sp}_{0}(2 n, \mathbb{R})$ is equivalent to $\operatorname{det}\left(P+Q-L-L^{T}\right) \neq 0$.

The value modulo 4 of $m+m^{\prime}-\mathrm{n}_{-}\left(P^{\prime}+Q\right)$ (recall the $\mathrm{n}_{-}(R)$ denotes the index of the symmetric matrix $R$ ) is independent of the choice of factorization, and thus only depends on $\widehat{\Phi}$ (see [8]):

Definition 5.3 The class modulo 4 of $m+m^{\prime}-\mathrm{n}_{-}\left(P^{\prime}+Q\right)$ is called the Maslov index of $\widehat{\Phi} \in \operatorname{Mp}(2 n, \mathbb{R})$; we denote it by $m(\widehat{\Phi})$. We call the function $m: \operatorname{Mp}(2 n, \mathbb{R}) \longrightarrow \mathbb{Z}_{4}$ thus defined "Maslov index" on $\operatorname{Mp}(2 n, \mathbb{R})$.

Let Inert be the 2-cocycle on $\Lambda$ defined by

$$
\operatorname{Inert}\left(L, L^{\prime}, L^{\prime \prime}\right)=\frac{1}{2}\left(\tau\left(L, L^{\prime}, L^{\prime \prime}\right)+n+\partial \operatorname{dim}\left(L, L^{\prime}, L^{\prime \prime}\right)\right.
$$

with $\partial \operatorname{dim}$ is the Čech coboundary of the 1-cochain $\operatorname{dim}\left(L, L^{\prime}\right)=\operatorname{dim}\left(L \cap L^{\prime}\right)$, that is

$$
\partial \operatorname{dim}\left(L, L^{\prime}, L^{\prime \prime}\right)=\operatorname{dim}\left(L \cap L^{\prime}\right)-\operatorname{dim}\left(L \cap L^{\prime \prime}\right)+\operatorname{dim}\left(L^{\prime} \cap L^{\prime \prime}\right) .
$$

We have (see [8]):

Proposition 5.4 We have $m\left(\widehat{\Phi}_{W, m}\right)=[m]_{4}$ and the Maslov index on $\operatorname{Mp}(2 n, \mathbb{R})$ is related to the Leray index $\mu$ by the formula

$$
m(\widehat{\Phi})=\left[\frac{1}{2}\left(\mu\left(\Phi_{\infty} L_{0, \infty}, L_{0, \infty}\right)+n+\operatorname{dim}\left(\Phi L_{0}, L_{0}\right)\right)\right]_{4}
$$

where $\Phi_{\infty}$ is any element of $\operatorname{Sp}_{\infty}(2 n, \mathbb{R})$ having projection $\widehat{\Phi} \in \operatorname{Mp}(2 n, \mathbb{R})$ and $L_{0, \infty}$ is any element of $\Lambda_{\infty}$ covering $L_{0}=0 \times \mathbb{R}^{n}$.

It follows from formula (16) and the properties of the Leray index that

$$
m\left(\widehat{\Phi} \widehat{\Phi}^{\prime}\right)=m(\widehat{\Phi})+m\left(\widehat{\Phi}^{\prime}\right)+\left[\operatorname{Inert}\left(L_{0}, \Phi L_{0}, \Phi \Phi^{\prime} L_{0}\right)\right]_{4} .
$$


5.2 Weyl representation and Conley-Zehnder index

Defining, as in [20], the operator $R_{v}(\phi)$ associated to $(\phi, v) \in \mathrm{Sp}_{0}(2 n, \mathbb{R}) \times \mathbb{Z}$ by the Bochner integral

$$
R_{\nu}(\phi)=\left(\frac{1}{2 \pi}\right)^{n} \frac{i^{\nu}}{\sqrt{|\operatorname{det}(\phi-\mathrm{Id})|}} \int e^{\frac{i}{2}\left\langle M_{\phi} z, z\right\rangle} T(z) d^{2 n} z
$$

where $T(z)$ is the Heisenberg-Weyl operator, we have (see [12], Proposition 6, Sect. 3.2 and Proposition 10, Sect. 3.3):

- Let $\phi_{W}$ be the free symplectic matrix generated by the quadratic form (14). We have $\widehat{\Phi}_{W, m}=R_{v}\left(\phi_{W}\right)$ if and only $v=v\left(\widehat{\Phi}_{W, m}\right)$ with

$$
v\left(\widehat{\Phi}_{W, m}\right) \equiv m-\mathrm{n}_{-}\left(W_{x x}\right) \bmod 4
$$

where $\mathrm{n}_{-}\left(W_{x x}\right)$ is the index of inertia of the Hessian matrix $W_{x x}$ of the function $x \longmapsto$ $W(x, x)$;

- Let $\widehat{\Phi} \in \operatorname{Mp}(2 n, \mathbb{R})$ be such that $\pi^{\mathrm{Mp}}(\widehat{\Phi}) \in \operatorname{Sp}_{0}(2 n, \mathbb{R})$. If $\phi=\phi_{W} \phi_{W^{\prime}}$ and

$$
\widehat{\Phi}=R_{v\left(\widehat{\Phi}_{W, m}\right)}\left(\phi_{W}\right) R_{v\left(\widehat{\Phi}_{W^{\prime}, m^{\prime}}\right)}\left(\phi_{W^{\prime}}\right)
$$

then $\widehat{\Phi}=R_{v(\widehat{\Phi})}(\phi)$ with $^{\star}$

$$
v(\widehat{\Phi}) \equiv v\left(\widehat{\Phi}_{W, m}\right)+v\left(\widehat{\Phi}_{W^{\prime}, m^{\prime}}\right)-\frac{1}{2} \operatorname{sign}\left(M_{\phi_{W}}+M_{\phi_{W}^{\prime}}\right) \quad \bmod 4 .
$$

These formulae suggest that there is a relation between the integer $v(\widehat{\Phi})$ and the ConleyZehnder index of some symplectic path ending at $\phi=\pi_{\mathrm{Mp}}(\widehat{\Phi})$. To study this relation we will need the following two lemmas:

Lemma 5.5 (i) Let $\left(L, L^{\prime}\right) \in(\Lambda(V, \omega))^{2}$. If $L \cap L^{\prime \prime}=0$ then $\tau\left(L, L^{\prime}, L^{\prime \prime}\right)$ is the signature of the quadratic form

$$
Q^{\prime}\left(z^{\prime}\right)=\omega\left(\operatorname{Pr}_{L L^{\prime \prime}} z^{\prime}, z^{\prime}\right)=\omega\left(z^{\prime}, \operatorname{Pr}_{L^{\prime \prime} L} z^{\prime}\right)
$$

on $L^{\prime}$, where $\operatorname{Pr}_{L L^{\prime \prime}}$ is the projection onto $L$ along $L^{\prime \prime}$ and $\operatorname{Pr}_{L^{\prime \prime} L}=\operatorname{Id}-\operatorname{Pr}_{L L^{\prime \prime}}$ is the projection on $L^{\prime \prime}$ along $L$. (ii) Let $\left(L, L^{\prime}, L^{\prime \prime}\right)$ be such that $L=L \cap L^{\prime}+L \cap L^{\prime \prime}$. Then $\tau\left(L, L^{\prime}, L^{\prime \prime}\right)=0$.

(See, e.g., [18] for a proof).

Lemma 5.6 Let $\Phi:[0,1] \longrightarrow \mathrm{Sp}(2 n, \mathbb{R})$ be a continuous path such that $\Phi(0)=\mathrm{Id}$, $\Phi(1)=\phi \in \operatorname{Sp}_{0}(2 n, \mathbb{R})$. Then

$$
\mathfrak{i}_{\mathrm{CZ}}(\Phi)=-\frac{1}{2} \mu^{2}\left((\operatorname{Id} \oplus \phi)_{\infty} \Delta_{\infty}, \Delta_{\infty}\right)
$$

where $\Delta_{\infty}$ is any element of $\Lambda_{\infty}\left(\mathbb{R}^{4 n}, \omega^{2}\right)$ with projection $\Delta=\left\{(z, z): z \in \mathbb{R}^{2 n}\right\}$ and $(\operatorname{Id} \oplus$ $\phi)_{\infty} \in \operatorname{Sp}_{\infty}\left(\mathbb{R}^{4 n}, \omega^{2}\right)$ is the homotopy class in $\operatorname{Sp}\left(\mathbb{R}^{4 n}, \omega^{2}\right)$ of the path $t \longmapsto\{(z, \Phi(t) z)$ : $\left.z \in \mathbb{R}^{2 n}\right\}, 0 \leq t \leq 1$.

Proof See [13].

It follows from the properties of the Leray index (see [9]) that the right-hand side of (20) does not depend on the choice of $\Delta_{\infty}$ covering $\Delta$.)

Let us now prove the main result of this section:

^ Recall that the Cayley transform used in this paper and that in [12] differ by a sign. 
Proposition 5.7 Let $\Phi_{\infty} \in \mathrm{Sp}_{\infty}(2 n, \mathbb{R})$ be the homotopy class of a continuous path $\Phi$ : $[0,1] \longrightarrow \operatorname{Sp}(2 n, \mathbb{R})$ such that $\Phi(0)=\mathrm{Id}, \Phi(1)=\phi \in \mathrm{Sp}_{0}(2 n, \mathbb{R})$. Let $\widehat{\Phi}$ the image of $\Phi_{\infty}$ in $\mathrm{Mp}(2 n, \mathbb{R})$ by the covering mapping $\operatorname{Sp}_{\infty}(2 n, \mathbb{R}) \longrightarrow \operatorname{Mp}(2 n, \mathbb{R})$. We have

$$
v(\widehat{\Phi}) \equiv-\mathfrak{i}_{\mathrm{CZ}}(\Phi) \bmod 4 \text {. }
$$

Proof Since $\widehat{\Phi}$ can be written as a product $\widehat{\Phi}_{W, m} \widehat{\Phi}_{W^{\prime}, m^{\prime}}$, formula (19) and the product formula (9) in Corollary 3.5 reduce the proof to the case $\widehat{\Phi}=\widehat{\Phi}_{W, m}$. In view of Lemma 5.6 and (18) it is sufficient to show that

$$
m-\mathrm{n}_{-}\left(W_{x x}\right) \equiv \frac{1}{2} \mu^{2}\left(\left(\operatorname{Id} \oplus \phi_{W}\right)_{\infty} \Delta_{\infty}, \Delta_{\infty}\right) \bmod 4 .
$$

We will divide the proof of (22) in three steps. We denote as before by $\omega^{2}$ the symplectic form $\omega \oplus(-\omega)$ on $\mathbb{R}^{4 n}=\mathbb{R}^{2 n} \oplus \mathbb{R}^{2 n}$; the corresponding Wall-Kashiwara and Leray indexes are $\tau^{2}$ and $\mu^{2}$.

(I) Let $L^{(2)} \in \Lambda\left(\mathbb{R}^{4 n}, \omega^{2}\right)$. Let $L_{\infty}^{(2)} \in \Lambda_{\infty}\left(\mathbb{R}^{4 n}, \omega^{2}\right)$ cover $L^{(2)} \in \Lambda\left(\mathbb{R}^{4 n}, \omega^{2}\right)$. Using the property $\partial \mu^{2}=\pi^{*} \tau^{2}$ we get after a few calculations

$$
\begin{aligned}
\mu^{2}\left(\left(\operatorname{Id} \oplus \phi_{W}\right)_{\infty} \Delta_{\infty}, \Delta_{\infty}\right)= & \left(\mu^{2}\left(\left(\operatorname{Id} \oplus \phi_{W}\right)_{\infty} L_{\infty}^{(2)}, L_{\infty}^{(2)}\right)\right. \\
& +\tau^{2}\left(\left(\operatorname{Id} \oplus \phi_{W}\right) \Delta, \Delta, L^{(2)}\right) \\
& \left.-\tau^{2}\left(\left(\operatorname{Id} \oplus \phi_{W}\right) \Delta,\left(\operatorname{Id} \oplus \phi_{W}\right) L^{(2)}, L^{(2)}\right)\right) .
\end{aligned}
$$

Choosing in particular $L^{(2)}=L_{0}^{(2)}=L_{0} \oplus L_{0}$ (recall: $L_{0}=\{0\} \times \mathbb{R}^{n}$ ) we get

$$
\begin{aligned}
\mu^{2}\left(\left(\operatorname{Id} \oplus \phi_{W}\right)_{\infty} L_{0, \infty}^{(2)}, L_{0, \infty}^{(2)}\right) & =\mu^{2}\left(\left(\operatorname{Id} \oplus \phi_{W}\right)_{\infty}\left(L_{0} \oplus L_{0}\right)_{\infty},\left(L_{0} \oplus L_{0}\right)_{\infty}\right) \\
& =\mu\left(L_{0, \infty}, L_{0, \infty}\right)-\mu\left(L_{0, \infty}, \Phi_{W, \infty} L_{P, \infty}\right) \\
& =\mu\left(\Phi_{W, \infty} L_{0, \infty}, L_{0, \infty}\right)
\end{aligned}
$$

so that there remains to prove that

$$
\tau^{2}\left(\left(\operatorname{Id} \oplus \phi_{W}\right) \Delta, \Delta, L_{0}^{(2)}\right)-\tau^{2}\left(\left(\operatorname{Id} \oplus \phi_{W}\right) \Delta,\left(\operatorname{Id} \oplus \phi_{W}\right) L_{0}^{(2)}, L_{0}^{(2)}\right)=-2 \operatorname{sign} W_{x x} .
$$

(II) We are going to show that $\tau^{2}\left(\left(\operatorname{Id} \oplus \phi_{W}\right) \Delta\right.$, (Id $\left.\left.\oplus \phi_{W}\right) L_{0}^{(2)}, L_{0}^{(2)}\right)=0$; in view of the symplectic invariance and the antisymmetry of $\tau^{2}$ this is equivalent to

$$
\tau^{2}\left(L_{0}^{(2)}, \Delta, L_{0}^{(2)},\left(\mathrm{Id} \oplus \phi_{W}\right)^{-1} L_{0}^{(2)}\right)=0 .
$$

We have

$$
\Delta \cap L_{0}^{(2)}=\left\{(0, p ; 0, p): p \in \mathbb{R}^{n}\right\}
$$

and $(\operatorname{Id} \oplus \phi)^{-1} L_{0}^{(2)} \cap L_{0}^{(2)}$ consists of all $\left(0, p^{\prime}, \phi^{-1}\left(0, p^{\prime \prime}\right)\right)$ with $\phi^{-1}\left(0, p^{\prime \prime}\right)=\left(0, p^{\prime}\right)$; since $\phi_{W}$ (and hence also $\phi_{W}^{-1}$ ) is free we must have $p^{\prime}=p^{\prime \prime}=0$ so that

$$
\left(\operatorname{Id} \oplus \phi_{W}\right)^{-1} L_{0}^{(2)} \cap L_{0}^{(2)}=\left\{(0, p ; 0,0): p \in \mathbb{R}^{n}\right\} .
$$

It follows that we have

$$
L_{0}=\Delta \cap L_{0}^{(2)}+\left(\operatorname{Id} \oplus \phi_{W}\right)^{-1} L_{0}^{(2)} \cap L_{0}^{(2)}
$$

hence (23) in view of property (ii) in Lemma 5.5. 
(III) Let us finally show that.

$$
\tau^{2}\left(\left(\operatorname{Id} \oplus \phi_{W}\right) \Delta, \Delta, L_{0}^{(2)}\right)=-2 \operatorname{sign} W_{x x} ;
$$

this will complete the proof. The condition $\operatorname{det}\left(\phi_{W}-\mathrm{Id}\right) \neq 0$ is equivalent to (Id $\left.\oplus \phi_{W}\right) \Delta \cap$ $\Delta=0$ hence, using property (i) in Lemma 5.5:

$$
\left.\tau^{2}\left(\left(\operatorname{Id} \oplus \phi_{W}\right) \Delta, \Delta, L_{0}^{(2)}\right)=-\tau^{2}\left(\operatorname{Id} \oplus \phi_{W}\right) \Delta, L_{0}^{(2)}, \Delta\right)
$$

is the signature of the quadratic form $Q$ on $L_{0}$ defined by

$$
Q\left(0, p, 0, p^{\prime}\right)=-\omega^{2}\left(\operatorname{Pr}_{\left(\operatorname{Id} \oplus \phi_{W}\right) \Delta, \Delta}\left(0, p, 0, p^{\prime}\right) ; 0, p, 0, p^{\prime}\right)
$$

where

$$
\operatorname{Pr}_{\left(\operatorname{Id} \oplus \phi_{W}\right) \Delta, \Delta}=\left[\begin{array}{cc}
\left(\phi_{W}-\mathrm{Id}\right)^{-1} & -\left(\phi_{W}-\mathrm{Id}\right)^{-1} \\
\phi_{W}\left(\phi_{W}-\mathrm{Id}\right)^{-1} & -\phi_{W}\left(\phi_{W}-\mathrm{Id}\right)^{-1}
\end{array}\right]
$$

is the projection on (Id $\left.\oplus \phi_{W}\right) \Delta$ along $\Delta$ in $\mathbb{R}^{2 n} \oplus \mathbb{R}^{2 n}$. It follows that the quadratic form $Q$ is given by

$$
Q\left(0, p, 0, p^{\prime}\right)=-\omega^{2}\left(\left(\operatorname{Id}-\phi_{W}\right)^{-1}\left(0, p^{\prime \prime}\right), \phi_{W}\left(\operatorname{Id}-\phi_{W}\right)^{-1}\left(0, p^{\prime \prime}\right) ; 0, p, 0, p^{\prime}\right)
$$

where we have set $p^{\prime \prime}=p-p^{\prime}$; by definition of $\omega^{2}$ this is

$$
Q\left(0, p, 0, p^{\prime}\right)=-\omega\left(\left(\operatorname{Id}-\phi_{W}\right)^{-1}\left(0, p^{\prime \prime}\right),(0, p)\right)+\omega\left(\phi_{W}(\operatorname{Id}-\phi)^{-1}\left(0, p^{\prime \prime}\right),\left(0, p^{\prime}\right)\right) .
$$

We have

$$
\left(\mathrm{Id}-\phi_{W}\right)^{-1}=J M_{\phi_{W}}+\frac{1}{2} \mathrm{Id}, \phi_{W}\left(\mathrm{Id}-\phi_{W}\right)^{-1}=J M_{\phi_{W}}-\frac{1}{2} \mathrm{Id}
$$

and hence

$$
\begin{aligned}
Q\left(0, p, 0, p^{\prime}\right) & =-\omega\left(\left(J M_{\phi_{W}}+\frac{1}{2} \mathrm{Id}\right)\left(0, p^{\prime \prime}\right),(0, p)\right)+\omega\left(\left(J M_{\phi_{W}}-\frac{1}{2} \mathrm{Id}\right)\left(0, p^{\prime \prime}\right),\left(0, p^{\prime}\right)\right) \\
& =-\omega\left(J M_{\phi_{W}}\left(0, p^{\prime \prime}\right),(0, p)\right)+\omega\left(J M^{\phi_{W}}\left(0, p^{\prime \prime}\right),\left(0, p^{\prime}\right)\right) \\
& =\omega\left(J M_{\phi_{W}}\left(0, p^{\prime \prime}\right),\left(0, p^{\prime \prime}\right)\right) \\
& =-\left\langle M_{\phi_{W}}\left(0, p^{\prime \prime}\right),\left(0, p^{\prime \prime}\right)\right\rangle .
\end{aligned}
$$

Let us calculate explicitly $M_{\phi_{W}}$. Writing $\phi_{W}=\left[\begin{array}{cc}A & B \\ C & D\end{array}\right]$ we have

$$
\phi_{W}-\mathrm{Id}=\left[\begin{array}{cc}
0 & B \\
\mathrm{Id} & D-\mathrm{Id}
\end{array}\right]\left[\begin{array}{cc}
C-(D-\mathrm{Id}) B^{-1}(A-\mathrm{Id}) & 0 \\
B^{-1}(A-\mathrm{Id}) & \mathrm{Id}
\end{array}\right]
$$

that is

$$
\phi_{W}-\mathrm{Id}=\left[\begin{array}{cc}
0 & B \\
\mathrm{Id} & D-\mathrm{Id}
\end{array}\right]\left[\begin{array}{cc}
W_{x x} & 0 \\
B^{-1}(A-\mathrm{Id}) & \mathrm{Id}
\end{array}\right]
$$

where we have used the identity

$$
\left.C-(D-\mathrm{Id}) B^{-1}(A-\mathrm{Id})\right)=B^{-1} A+D B^{-1}-B^{-1}-\left(B^{T}\right)^{-1}
$$


which follows from the relation $C-D B^{-1} A=-\left(B^{T}\right)^{-1}$ due to the fact that $\phi_{W}$ is symplectic. We thus have, writing $W_{x x}^{-1}=\left(W_{x x}\right)^{-1}$,

$$
\begin{aligned}
\left(\phi_{W}-\mathrm{Id}\right)^{-1} & =\left[\begin{array}{cc}
W_{x x}^{-1} & 0 \\
B^{-1}(\operatorname{Id}-A) W_{x x}^{-1} & \mathrm{Id}
\end{array}\right]\left[\begin{array}{cc}
(\mathrm{Id}-D) B^{-1} & \mathrm{Id} \\
B^{-1} & 0
\end{array}\right] \\
& =\left[\begin{array}{cc}
W_{x x}^{-1}(\mathrm{Id}-D) B^{-1} & W_{x x}^{-1} \\
B^{-1}(\operatorname{Id}-A) W_{x x}^{-1}(\mathrm{Id}-D) B^{-1}+B^{-1} & B^{-1}(\operatorname{Id}-A) W_{x x}^{-1}
\end{array}\right]
\end{aligned}
$$

and hence

$$
M_{\phi_{W}}=\left[\begin{array}{cc}
B^{-1}(\operatorname{Id}-A) W_{x x}^{-1}(\operatorname{Id}-D) B^{-1}+B^{-1} & \frac{1}{2} \operatorname{Id}+B^{-1}(\operatorname{Id}-A) W_{x x}^{-1} \\
-\frac{1}{2} \operatorname{Id}-W_{x x}^{-1}(\operatorname{Id}-D) B^{-1} & -W_{x x}^{-1}
\end{array}\right]
$$

so that we have

$$
Q\left(0, p, 0, p^{\prime}\right)=\left\langle W_{x x}^{-1} p^{\prime \prime}, p^{\prime \prime}\right\rangle=\left\langle W_{x x}^{-1}\left(p-p^{\prime}\right),\left(p-p^{\prime}\right)\right\rangle .
$$

It follows that the matrix of the quadratic form $Q$ is

$$
2\left[\begin{array}{cc}
W_{x x}^{-1} & -W_{x x}^{-1} \\
-W_{x x}^{-1} & W_{x x}^{-1}
\end{array}\right]
$$

and this matrix has signature $2 \operatorname{sign}\left(W_{x x}\right)^{-1}=2 \operatorname{sign} W_{x x}$. This concludes the proof since $\operatorname{det}\left(\phi_{W}-\mathrm{Id}\right)=(-1)^{n} \operatorname{det} B \operatorname{det} W_{x x}$ and $2 m-m=\mu\left(\phi_{W, \infty} L_{0, \infty}, L_{0, \infty}\right)$.

Acknowledgements The first author (MdG) has been financed by a FAPESP grant, and he wishes to thank all faculties and staff at the University of São Paulo for providing excellent working conditions during his stay.

\section{References}

1. Cappell, S.E., Lee, R., Miller, E.Y.: On the Maslov index. Comm. Pure Appl. Math. 47(2), 121-186(1994)

2. Cappell, S.E., Lee, R., Miller, E.Y.: Self-adjoint elliptic operators and manifold decompositions, Part I: Low eigenmodes and stretching. Comm. Pure Appl. Math. 49, 825-866 (1996)

3. Cappell, S.E., Lee, R., Miller, E.Y.: Self-adjoint elliptic operators and manifold decompositions, Part II: Spectral Flow and Maslov. index. Comm. Pure Appl. Math. 49, 869-909 (1996)

4. Conley, C.C., Zehnder, E.: Morse-type index theory for flows and periodic solutions of Hamiltonians. Comm. Pure Appl. Math. 37, 207-253 (1984)

5. Cushman, R., Duistermaat, J.J.: The behavior of the index of a periodic linear Hamiltonian system under iteration. Adv. Math. 23(1), 1-21 (1977)

6. Duistermaat, J.J.: On the Morse index in variational calculus. Adv. Math. 21(2), 173-195 (1976)

7. Dazord, P.: Invariants homotopiques attachés aux fibrés symplectiques. Ann. Inst. Fourier 29(2), 2578 (1979)

8. de Gosson, M.: Maslov indices on the metaplectic group Mp(n). Ann. Inst. Fourier 40(3), 537-555 (1990)

9. de Gosson, M.: The structure of $q$-symplectic geometry. J. Math. Pures Appl. 71, 429-453 (1992)

10. de Gosson, M.: Maslov Classes, Metaplectic Representation and Lagrangian Quantization. Research Notes in Mathematics, vol. 95, Berlin, Wiley-VCH (1997)

11. de Gosson, M.: Lagrangian path intersections and the Leray index. In: Geometry and topology: Aarhus (1998), pp. 177-184. Contemp. Math., vol. 258. Providence, RI, Amer. Math. Soc (2000)

12. de Gosson, M.: On the Weyl representation of metaplectic operators. Lett. Math. Phys. 72, 129142 (2005)

13. de Gosson, S.: Multi-oriented symplectic geometry and the extension of path intersection indices. $\mathrm{PhD}$ thesis, Växjö University, http://www.diva-portal.org/vxu/abstract.xsql?dbid=400 (2005)

14. Duistermaat, J.J.: On the Morse index in variational calculus. Adv. Math. 21, 173-195 (1976) 
15. Gutzwiller, M.C.: Chaos in classical and quantum mechanics. Interdisciplinary Applied Mathematics, Springer-Verlag (1990)

16. Hofer, H., Wysocki, K., Zehnder, E.: Properties of pseudoholomorphic curves in symplectizations II: Embedding controls and algebraic invariants. Geom. Funct. Anal. 2(5), 270-328 (1995)

17. Hörmander, L.: Fourier integral operators. Acta Math. 127, 79-183 (1971)

18. Lion, G., Vergne, M.: The Weil representation, Maslov index and Theta series. Prog. Math. Vol. 6, Birkhäuser, Boston (1980)

19. Long, Y.: Precise iteration formulae of the Maslov-type index theory and ellipticity of closed characteristics. Adv. Math. 154, 76-131 (2000)

20. Mehlig, B., Wilkinson, M.: Semiclassical trace formulae using coherent states. Ann. Phys. 10(6-7), 541555 (2001)

21. Morvan, J.-M.: Maslov, Duistermaat, Conley-Zehnder invariants in Riemannian Geometry. In: Geometry and Topology of Submanifold, V (Leuven/Brussels, 1992), pp. 174-200. World Sci. Publishing, River Edge, NJ (1993)

22. Muratore-Ginanneschi, P.: Path integration over closed loops and Gutzwiller's trace formula. Phys. Rep. 383, 299-397 (2003)

23. Robbin, J., Salamon, D.: The Maslov index for paths. Topology 32(4), 827-844 (1993)

24. Robbin, J., Salamon, D.: The spectral flow and the maslov index. Bull. London Math. Soc. 27, 1-33 (1995)

25. Salamon, D., Zehnder, E.: Morse theory for periodic solutions of Hamiltonian systems and the Maslov index. Comm. Pure Appl. Math. 45, 1303-1360 (1992)

26. Wall, C.T.C.: Nonadditivity of the signature. Invent. Math. 7, 269-274 (1969) 